\title{
Lung Papillary Adenocarcinoma
}

National Cancer Institute

\section{Source}

National Cancer Institute. Lung Papillary Adenocarcinoma. NCI Thesaurus. Code C5650.

A morphologic variant of lung adenocarcinoma characterized by the presence of papillary structures. 